\title{
Taxa metabólica de repouso e composição corporal em mulheres na pós-menopausa
}

\author{
Resting metabolic rate and body composition in postmenopausal women
}

Valéria Bonganha' ', Miguel Soares Conceição', Claudinei Ferreira dos Santos' Mara Patrícia Traína Chacon-Mikahil', Vera Aparecida Madruga'

\begin{abstract}
RESUMO
Objetivo: A presente pesquisa avaliou a relação entre a taxa metabólica de repouso (TMR) e a composição corporal de mulheres na pós-menopausa. Métodos: Participaram 30 mulheres inativas fisicamente, com média de idade $54,33 \pm 5,20$ anos. 0 consumo de oxigênio foi avaliado por meio de calorimetria indireta após 12 horas em jejum e os valores obtidos foram calculados segundo a equação de Weir. A composição corporal foi obtida pelo método de dobras cutâneas e a medida da circunferência de cintura (CC) foi utilizada para avaliar a gordura abdominal. Utilizou-se a correlação linear de Pearson para estabelecer as correlações entre as variáveis. Resultados: Foram encontradas correlações significantes da TMR com a CC $(r=0,42)$ e com a massa magra (MM) $(r=0,48)$. Conclusões: As variáveis da composição corporal que podem estar envolvidas na determinação daTMR são a MM e a CC. Arq Bras Endocrinol Metab. 2009;53(6):755-9

Descritores

Envelhecimento; menopausa; composição corporal; adiposidade; metabolismo energético
\end{abstract}

\section{ABSTRACT}

Objective: The present study evaluated the relationship between resting metabolic rate (RMR) and body composition of postmenopausal women. Methods: Thirty physically inactive women participated in the study, and their age average was 54,33 $\pm 5,20$ years old. Oxygen consumption was measured by indirect calorimetry after 12 hours of fasting and the values were calculated according to the equation of Weir. Body composition was obtained by the method of skinfolds and the measurement of waist circumference (WC) was used to assess abdominal fat. The linear correlation of Pearson was used to establish correlations between the variables. Results: We found significant correlations ofTMR with the CC (0.42) and the lean mass (LM) ( $r=$ 0.48). Conclusions: The variables of body composition that can be involved in the determination of the RMR are LM and WC. Arq Bras Endocrinol Metab. 2009;53(6):755-9

Keywords

Aging; menopause; body composition; adiposity; energy metabolism

\section{INTRODUÇÃO}

$\mathrm{O}$ avanço da idade tem sido associado ao declínio na atividade física e, consequentemente, a uma redução da massa magra (MM), do gasto energético e aumento da massa gorda $(\mathrm{MG})$, fatores que influenciam a diminuição da taxa metabólica de repouso (TMR) e que contribuem para o aumento do peso corporal, principalmente nas mulheres na pós-menopausa (1).

A taxa mínima de energia consumida para manter as funções fisiológicas no estado de repouso é conhecida como a TMR (2). Nessas condições, não estão incluídos
${ }^{1}$ Laboratório de Fisiologia do Exercício (FISEX), Faculdade de Educação Física, Universidade Estadual de Campinas (FEF/ Unicamp), Campinas, SP, Brasil

\author{
Correspondência para: \\ Valéria Bonganha \\ Laboratório de Fisiologia do \\ Exercício da Unicamp \\ Av. Érico Veríssimo, 701 - Cidade \\ Universitária, Barão Geraldo \\ 13083-851 - CP 6134, Campinas, \\ SP, Brasil \\ valeriabonganha@hotmail.com
}

Recebido em 5/Fev/2009 Aceito em 26/Mai/2009 os efeitos térmicos dos alimentos, ou seja, o período em que os macronutrientes ainda estão sendo absorvidos pelos tecidos. Esse período pode durar cerca de 10 a 12 horas após a última refeição $(3,4)$.

A TMR representa o principal componente do gasto energético diário, correspondendo a $60 \%$ a $75 \%$ do gasto total. Os outros componentes do gasto energético diário são o efeito térmico dos alimentos, correspondendo a aproximadamente $10 \%$, e gasto energético com atividade física (agudo ou crônico), entre 15\% a $30 \%(5)$. 
A determinação da TMR depende, em grande parte, da quantidade de MM e da atividade dos tecidos metabolicamente ativos, como coração, cérebro, rins e fígado (6).

Existe uma correlação inversa entre a idade e a TMR. Essa relação é atribuída a fatores como a quantidade diminuída de MM e concomitante aumento da MG, conteúdos de fluidos corporais, alterações na temperatura corporal, alterações hormonais, área corporal total, inatividade física e fatores genéticos (7).

O decréscimo da TMR com o aumento da idade, especificamente nas mulheres, também pode ser resultado das alterações na composição corporal causadas pela menopausa (8). A partir dos 20 anos, as mulheres têm declínio da TMR de cerca de $2 \%$ por década e a diminuição da MM tem influência direta nesse declínio (9). Entre essas alterações, destacam-se: a redução da MM, o aumento e redistribuição da gordura corporal, e subsequente aumento da massa corporal total (MCT) $(10,11)$. As alterações na densidade mineral óssea e o uso de terapias de reposição hormonal (TRH) também possuem correlações fortes com a TMR $(4,8,12,13)$.

A fase da menopausa parece contribuir de maneira mais significativa para o decréscimo da TMR do que o processo de envelhecimento por si só em razão da diminuição dos níveis de hormônios femininos (4). Entre suas diversas funções, destacam-se a atuação sobre o substrato energético no repouso e durante o exercício, a regulação do metabolismo de carboidratos e lipídios e a participação no processo de síntese de proteínas $(14,15)$.

Embora seja atribuída somente à MM uma função metabolicamente ativa, há investigações sobre efeitos semelhantes para a MG. Sendo assim, a estimativa da TMR pode ser influenciada tanto pela quantidade de MM quanto de gordura corporal (16), sugerindo que a localização da gordura corporal, principalmente na região abdominal, possui maior importância na determinação da estimativa da TMR do que a quantidade de gordura total (16).

Nesse sentido, a circunferência da cintura (CC) é um indicador simples e fidedigno da presença de gordura abdominal (17), sendo preditor de doenças metabólicas e risco de obesidade independentemente do índice de massa corporal (IMC) e da gordura corporal total $(11,18)$.

O aumento dos valores da TMR na fase de envelhecimento, por meio de mudanças na composição corporal, pode ser medida de manutenção ou prevenção contra o aumento de MCT, colaborando para a dimi- nuição de incidência de doenças crônico-degenerativas, geralmente agravadas com o início da menopausa nas mulheres $(1,3,19)$.

Dessa maneira, o objetivo do presente estudo foi relacionar a TMR com as variáveis da composição em mulheres na pós-menopausa.

\section{MÉTODOS}

Neste estudo transversal participaram 30 mulheres na pós-menopausa, clinicamente saudáveis, inativas fisicamente, sem uso de terapia de reposição hormonal e com média de idade 54,3 $\pm 5,2$ anos. Como critérios iniciais de inclusão, as voluntárias deveriam estar no período da pós-menopausa (ausência mínima de 12 meses de menstruações) e não ser ativas fisicamente (frequência de atividade física regular menor que duas sessões por semana).

Os critérios de exclusão foram: cardiopatias graves, obesidade mórbida, doenças metabólicas e uso de medicamentos que pudessem interferir nos resultados. Para tanto, as voluntárias foram submetidas a exames clínicos realizados por um médico cardiologista no Hospital das Clínicas da Universidade Estadual de Campinas (Unicamp).

Todas as voluntárias, após serem conscientizadas sobre a proposta do estudo e procedimentos aos quais seriam submetidas, assinaram o termo de consentimento livre e esclarecido.

A presente pesquisa foi aprovada pelo Comitê de Ética em Pesquisa da Faculdade de Ciências Médicas da Unicamp (parecer CEP-FCM/Unicamp n ${ }^{\circ} 248 / 2004$, com adendo em 2007).

\section{Avaliação antropométrica e composição corporal}

A MCT foi mensurada numa balança de plataforma tipo Filizolla, e a medida da estatura foi obtida por um estadiômetro de madeira. A medida da circunferência de cintura (CC) foi utilizada para avaliar a gordura abdominal.

A composição corporal foi obtida pela técnica de mensuração das espessuras das dobras cutâneas. Para tanto, essas dobras foram aferidas na região peitoral, abdominal, coxa, subescapular, tricipital, bicipital, axilar média, suprailíaca e panturrilha média. Tais medidas foram coletadas com a utilização de um adipômetro calibrado, da marca LANGE$^{\circledR}$.

Todos os procedimentos da avaliação antropométrica e da composição corporal foram realizados de acordo com as técnicas descritas por Lohman (20). 
A densidade corporal foi obtida por meio da equação de Jackson e Pollock (21) e o percentual (\%) de gordura, pela fórmula de Siri (22). Para o cálculo da $\mathrm{MG}$, foi utilizada a seguinte fórmula: $\mathrm{MG}(\mathrm{kg})=\mathrm{MCT}$ x (\%gordura/100). A MM foi estimada pelo cálculo: $\mathrm{MM}(\mathrm{kg})=\mathrm{MCT}-\mathrm{MG}$.

\section{Avaliação da taxa metabólica de repouso}

Para a avaliação da TMR, foram coletados valores da calorimetria indireta $\left(\mathrm{O}_{2}\right.$ e $\left.\mathrm{CO}_{2}\right)$ (Ultima CPX, MedGraphics USA) com cálculo realizado por meio da equação de Weir (23).

O teste foi realizado em ambiente de condições laboratoriais, durante 30 minutos, coletando-se respiração a respiração. Para a computação dos dados, foram desprezados os dez minutos iniciais para a estabilidade das variáveis fisiológicas no repouso.

Para esse teste, as voluntárias ficaram em posição supina, em silêncio absoluto, evitando se mexerem, sendo que não era permitido dormir. Todas usaram uma máscara conectada ao analisador de gases, devidamente calibrado antes de cada teste.

As voluntárias permaneceram em jejum nas 12 horas antecedentes ao teste. Também foram orientadas a não realizarem exercícios físicos, ingerir bebidas cafeinadas ou alcoólicas nas 24 horas antecedentes ao teste. As voluntárias eram orientadas a fazer o mínimo de esforço físico para chegar ao local da avaliação. Essas avaliações foram realizadas entre 7 e 9 horas da manhã.

$\mathrm{O}$ gasto energético diário $(\mathrm{kcal} / \mathrm{dia})$ foi calculado pela equação de Weir $(23)$ :

Equação $=\left[\left(3,9^{*} \mathrm{O}_{2}\right)+\left(1,1^{*} \mathrm{CO}_{2}\right)^{*} 1440\right.$, onde: $\mathrm{O}_{2}$ é o valor médio consumido (litros/minuto) e $\mathrm{CO}_{2}$ é o valor médio expirado (litros/minuto) durante os 20 minutos computados para a análise.

\section{Análise estatística}

Para a análise dos dados, verificou-se inicialmente a normalidade da amostra pelo teste de Shapiro-Wilks. A estatística descritiva foi utilizada para calcular os valores médios e o desvio-padrão de todas as variáveis analisadas. O teste de correlação linear de Pearson foi usado para estabelecer as correlações existentes entre a TMR, a idade e as variáveis da composição corporal. Também se calculou o coeficiente de determinação $\left(\mathrm{r}^{2} \mathrm{x} 100\right)$.

O nível de significância adotado foi de $\mathrm{p}<0,05$. Para todas as análises, utilizou-se o programa estatístico SPSS 10.0.

\section{RESULTADOS}

Características antropométricas e composição corporal da amostra estudada estão apresentadas na tabela 1 .

Os valores médios encontrados para a taxa metabólica de repouso estão apresentados na tabela 2 .

A tabela 3 aponta correlações significantes entre a TMR com a MM $(r=0,481)$ e com a CC $(r=0,417)$. Entretanto, para as outras variáveis não foram encontrados resultados significantes que remetessem a alguma relação expressiva entre elas.

\begin{tabular}{lc}
\hline $\begin{array}{l}\text { Tabela 1. Características antropométricas e composição corporal da amostra } \\
\text { estudada }\end{array}$ \\
\hline Variáveis & Média $\pm \mathbf{~ d p ~}$ \\
\hline Estatura $(\mathrm{m})$ & $1,57 \pm 0,05$ \\
MCT $(\mathrm{kg})$ & $64,30 \pm 8,48$ \\
IMC $\left(\mathrm{kg} / \mathrm{m}^{2}\right)$ & $25,87 \pm 2,77$ \\
CC $(\mathrm{cm})$ & $80,41 \pm 8,34$ \\
Gordura (\%) & $34,02 \pm 6,39$ \\
$\Sigma 9 D C$ & $225,23 \pm 42,43$ \\
MM (kg) & $43,01 \pm 4,38$ \\
MG $(\mathrm{kg})$ & $21,10 \pm 5,23$ \\
\hline
\end{tabular}

dp: desvio-padrão

\begin{tabular}{lc}
\hline \multicolumn{2}{l}{ Tabela 2. Valores médios para a taxa metabólica de repouso } \\
\hline TMR & Média $\pm \mathbf{d p}$ \\
\hline TMR (kcal/dia) & $1048,02 \pm 146,24$ \\
TMR (kcal/h) & $179,08 \pm 24,78$ \\
TMR (kcal/MCT/h) & $2,81 \pm 0,39$ \\
TMR (kcal/MM/h) & $4,17 \pm 0,5$ \\
\hline
\end{tabular}

dp: desvio-padrão.

\begin{tabular}{|c|c|c|c|}
\hline \multirow{2}{*}{ Variáveis } & \multirow{2}{*}{$\begin{array}{c}\text { Correlação (r) } \\
\text { TMR }\end{array}$} & \multirow{2}{*}{ p } & \multirow{2}{*}{$\begin{array}{c}\text { Coeficiente de } \\
\text { determinação } \\
\left(\mathbf{r}^{2}\right)(\%)\end{array}$} \\
\hline & & & \\
\hline Idade (anos) & $-0,210$ & 0,265 & 4,41 \\
\hline MCT (kg) & 0,353 & 0,056 & 12,46 \\
\hline IMC (kg) & 0,254 & 0,175 & 6,45 \\
\hline CC (cm) & $0,417^{\star}$ & 0,02 & 17,38 \\
\hline MM (kg) & $0,481^{*}$ & 0,007 & 23,13 \\
\hline$M G(k g)$ & 0,160 & 0,399 & 2,56 \\
\hline$\Sigma 9 D C$ & $-0,09$ & 0,633 & 0,81 \\
\hline$\%$ Gordura & 0,01 & 0,998 & 0,001 \\
\hline
\end{tabular}

* Representa diferença estatisticamente significativa $(p<0,05)$. dp: desvio-padrão. 


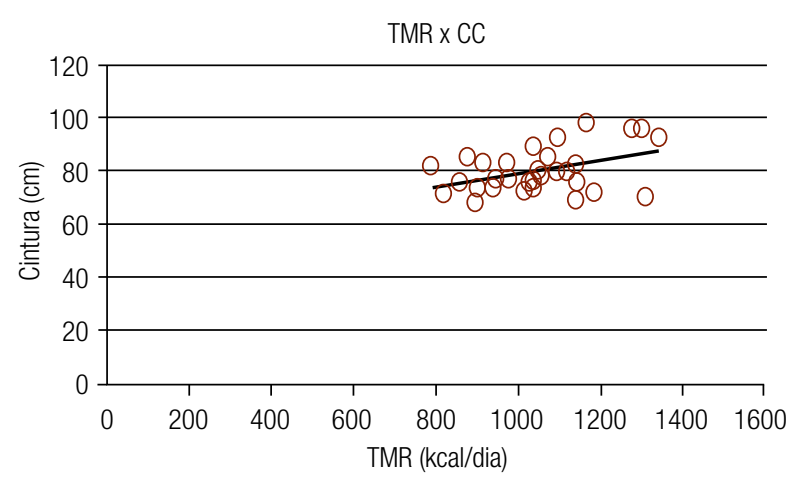

$r=0,42 ; p<0,02$

Figura 1. Correlação linear de Pearson entre a taxa metabólica de repouso (TMR) e a circunferência de cintura (CC).

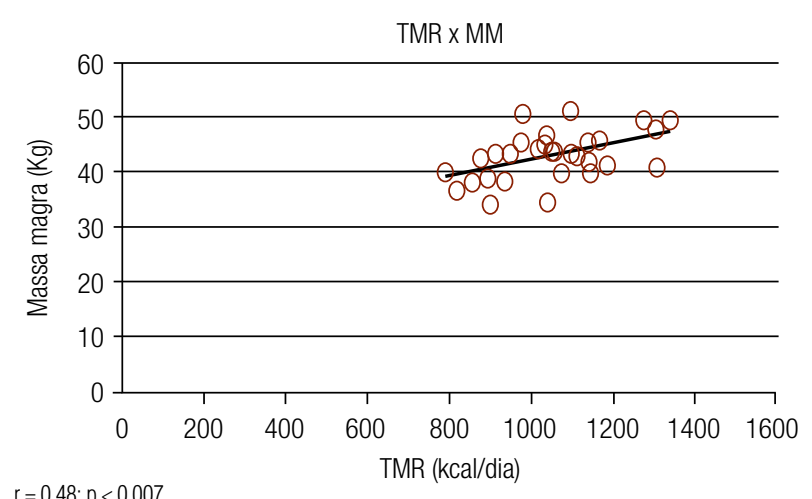

$r=0,48 ; p<0,007$

Figura 2. Correlação linear de Pearson entre a taxa metabólica de repouso (TMR) e a massa magra (MM).

\section{DISCUSSÃO}

A partir da equação elaborada por Weir, em 1949, estudos sobre TMR têm sido realizados $(7,24-27)$, contudo, as variáveis da composição corporal determinantes para a TMR ainda não estão completamente claras.

A associação encontrada entre a TMR e a idade $(r=$ $-0,210)$ não nos remete a nenhuma relação expressiva, como mencionado na literatura (7), provavelmente pela pequena amplitude da faixa etária da amostra avaliada.

A perda da MM é uma mudança decorrente das alterações hormonais da menopausa e que se acentuam com o envelhecimento. Encontrou-se correlação positiva entre a MM e a TMR, e essas afirmações podem ser reforçadas de acordo com os resultados de outros estudos (24).

A quantidade de MM e sua relação com a estimativa da TMR são explicadas por se tratar de um tecido metabolicamente ativo que necessita de grande síntese proteica (28). Contudo, há investigações sobre efeitos semelhantes para a MG (16).

Os resultados encontrados mostraram correlação significativa entre a CC e a TMR $(r=0,42)$, indicando que a gordura abdominal pode ter influência na predição da TMR, visto que outros indicadores de adiposi- dade, como porcentagem de gordura, MG e soma de dobras cutâneas, não apresentaram correlações significantes com a TMR. Entretanto, as relações da TMR com a CC ainda não estão bem elucidadas $(6,16,27)$, e os resultados apresentados no presente estudo são apenas indícios de tal comportamento.

Corroborando esses achados, o estudo de Luhrmann (16) buscou avaliar as relações da gordura abdominal e periférica com a TMR, mas os resultados mostraram que somente a gordura localizada na região abdominal teve influência sobre a TMR em mulheres.

Um estudo não muito recente (22) mostrou que a MG é também um importante preditor da TMR, explicando $2 \%$ a $3 \%$ da variabilidade de sua estimativa. Esses resultados também confirmam as observações do estudo de Tataranni (27), realizado com indivíduos jovens e de meia-idade, cujos resultados mostraram que a $\mathrm{MG}$ corresponde entre $1 \%$ e $10 \%$ da variabilidade da TMR, corroborando o resultado aqui verificado $(11,28)$.

Confrontando os achados da literatura mencionados acima, infere-se que a CC possui um papel com maior evidência do que a MG e a MCT na predição da TMR.

$O$ padrão de distribuição da gordura corporal tem mostrado maior importância do que a quantidade de gordura corporal total para o risco de distúrbios metabólicos e doenças cardiovasculares $(18,28)$. Medidas de CC maiores ou iguais a $88 \mathrm{~cm}$ para mulheres e 102 $\mathrm{cm}$ para homens são consideradas fator de risco elevado para doenças cardiovasculares (29).

A gordura abdominal/visceral é metabolicamente mais ativa do que as demais, liberando grandes quantidades de ácidos graxos livres na circulação portal, reduzindo a extração hepática de insulina, ocasionando hiperinsulinemia periférica (28). Além disso, é marcada por um maior fluxo sanguíneo, maior responsividade à noradrenalina e menor sensibilidade ao efeito antilipolítico da insulina. Também exerce maior atividade simpática do sistema nervoso e um aumento da taxa de lipólise quando em comparação ao tecido adiposo subcutâneo (30).

Tais características desempenham um papel importante no desenvolvimento de várias complicações metabólicas (26), além de aparentemente explicarem a correlação positiva existente entre a gordura central e a TMR.

Com as alterações na composição corporal está um agravante que, além de reforçar as mudanças da menopausa, contribui para a diminuição da TMR com o envelhecimento: a inatividade física (1). Estudos apontam que a inatividade física está presente na maioria da população em processo de envelhecimento, ocasionan- 
do maior aparecimento de doenças, comprometendo o estado geral de saúde $(19,30)$.

A estimativa da TMR pode ajudar na elaboração de estratégias para reverter mudanças oriundas da menopausa e envelhecimento, pois é um indicador indireto do balanço energético. Quando bem elaboradas, essas estratégias podem resultar em ganho ou redução da MCT, relacionados à mortalidade e à morbidade de indivíduos idosos (3).

Sugere-se a realização de novos estudos que utilizem avaliações diretas (resistência à insulina, níveis de colesterol e triglicerídeos) para identificar a atividade metabólica da gordura visceral na população em fase de envelhecimento.

Conclui-se, por meio dos resultados encontrados, que a estimativa da TMR pode ser influenciada tanto pela MM como pela CC. Entretanto, os mecanismos envolvidos na relação da CC com a TMR ainda não estão esclarecidos e, notoriamente, essa relação não é benéfica, tendo em vista a série de complicações metabólicas provocadas pela gordura abdominal.

Agradecimentos: aos pós-graduandos Giovana Vergínia de Souza e Cleiton Augusto Libardi, à graduanda Nathália Volpato pelas contribuições nas coletas de dados e à Renata Maria de Oliveira Botelho pela ajuda em todo o processo. À Coordenação de Aperfeiçoamento de Pessoal de Nível Superior (Capes) pela Bolsa Pesquisa de Mestrado.

Declaração: os autores declaram não haver conflitos de interesse científico neste estudo.

\section{REFERÊNCIAS}

1. Hallal PC, Victora CG, Wells JC, Lima RC. Physical inactivity: prevalence and associated variables in Brazilian adults. Med Sci Sports Exerc. 2003;35(11):1894-900.

2. Melby C, Scholl C, Edwards G, Bullough R. Effects of acute resistance on post-exercise energy expenditure and resting metabolic rate. J Appl Physiol. 1993;75(4):1847-53.

3. Leal DB, Fonseca PHS. Implicações na medida de taxa metabólica de repouso em idosos. Rev Bras Nutr Clin. 2007;22(1):65-71.

4. Aubertin-Leheudre M, Goulet EDB, Dionne IJ. Enhanced rate of resting energy expenditure in women using hormone replacement therapy: preliminary results. J Aging Physi Activ. 2008;16(1):53-60.

5. Foreaux G, Pinto KMC, Dâmaso A. Efeito do consumo excessivo de oxigênio após exercício e da taxa metabólica de repouso no gasto energético. Rev Bras Med Esporte. 2006;12(6):393-8.

6. Nelson KM, Weinsier RL, Long CL, Schutz Y. Prediction of resting energy expenditure from fat-free mass and fat mass. Am J Clin Nutr. 1992;56(5):848-56.

7. Antunes HKM, Santos RF, Boscolo RA, Bueno OFA, Mello MT. Análise da taxa metabólica basal de idosos do sexo masculino antes e seis meses após exercícios de resistência. Rev Bras Med Esporte. 2005;11(1):71-5.

8. Day DS, Gozansky WS, Van Pelt RE, Schwarts RS, Kohrt WM. Sex hormones suppression reduces resting energy expenditure and $\beta$-adrenergic support of resting energy expenditure. J Clin Endocrinol Metabolism. 2005;90(6):3312-7.
9. Armellini F, Zamboni M, Mino A, Bissoli L, Micciolo R, Bosello O. Postabsorptive resting metabolic rate and thermic effect of food in relation to body composition and adipose tissue distribution. Metabolism. 2000;149(1):6-10.

10. Sternfeld B, Bhat AK, Wang H, Sharp T, Quesenberry CP. Menopause, physical activity, and body composition/fat distribution in midlife women. Med Sci Sports Exerc. 2005;37(7):1195-202.

11. Janssen I, Heymsfield SB, Ross R. Low relative skeletal muscle mass (sarcopenia) in older persons is associated with functional impairment and physical disability. J Am Geriatr Soc. 2002;50(5):889-96.

12. Choi JW, Pai SH. Bone mineral density correlates strongly with basal metabolic rate in postmenopausal women. Clin Chimica Acta. 2003;333(1):79-84.

13. Anderson EJ, Lavoio HB, Strauss CC, Hubbard JL, Sharpless JL, Hall JE. Body composition and energy balance: lack of effects of short-term hormone replacement in postmenopausal women. Metabolism. 2001;50(3):265-9.

14. Lemoine S, Granier P,Tiffoche C, Rannou-Bekono F, Thieulant ML, Delamarche P. Estrogen receptor alpha mRNA in human skeletal muscles. Med Sci Sports Exerc. 2003;35(3):439-43.

15. Hackney AC, Muoio D, Meyer WR. The effect of sex steroids hormones on substrate oxidationduring prolonged submaximal exercise in women. Jpn J Physiol. 2000;50(5):489-94.

16. Luhrmann PM, Herbert BM, Neuhauser-Berthold M. Effects of fat mass and body composition on resting metabolic rate in the elderly. Metabolism. 2001;50(8):972-5.

17. Rankinen T, Kim SY, Pérusse L, Després J-P, Bouchard C. The prediction of abdominal visceral fat level from body composition and anthropometry: ROC analysis. Int J Obes. 1999;23(8):801-9.

18. Wong SL, Janssen $L$, Ross R. Abdominal adipose tissue distribution and metabolic risk. Sports Med. 2003;33(10):709-26.

19. Gibbons MDR, Henry CJK, Ulijaszek SJ, Lightowler HJ. Intra-individual variation in RMR in older people. $\mathrm{Br} \mathrm{J}$ Nutr. 2004;91(3):485-9.

20. Lohman TG, Roche AF, Martorell R, editors. Anthropometric standardizing reference manual. Champaign, Illinois: Human Kinetics Books; 1988. p. 55-80.

21. Jackson AS, Pollock ML. Generalized equations for predicting body density of women. Med Sci Sports Exerc. 1980;12(3):175-82.

22. Siri WE. Body composition from fluid spaces and density. In: Brozek J, Henschel A. Techniques for measuring body composition. Washington, DC: National Academy of Science; 1961. p. 223-44.

23. Weir JB. New methods for calculating metabolic rate with especial references to protein metabolism. J Physiol. 1949;62:20-7.

24. Trevisan MC, Burini RC. Metabolismo de repouso de mulheres pós-menopausadas submetidas a programa de treinamento com pesos (hipertrofia). Rev Bras Med Esporte. 2007;13(2):133-7.

25. Sparti A, DeLany JP, Bretonne JA, Sander GE, Bray GA. Relationship between resting metabolic rate and the composition of the fat-free mass. Metabolism. 1997;46(10):1225-30.

26. Bouchard C, Bray GA, Hubbard VS. Basic and clinical aspects of regional fat distribution. Am J Clin Nutr. 1990;52(5):946-50.

27. Tataranni PA, Ravussin E. Variability in metabolic rate: biological sites of regulation. Int J Obes. 1995;19(Suppl 4):S102-6.

28. Kabir M, Catalano KJ, Ananthnarayan S, Kim SP, Van Citters GW, Dea Mk, et al. Molecular evidence supporting the portal theory: a causative link between visceral adiposity and hepatic insulin resistance. Am J Physiol Endocrinol Metab. 2005;288(2):454-61.

29. National Institute of Health.Third report of the National Institute Cholesterol Education Program Expert Panel on detection, evaluation, and treatment of high blood cholesterol in adults (Adult Treatment Panel III). Executive Summary. NIH Publications. 2001;(1):3670.

30. Macera CA, Jones DA, Yore MM, Ham SA, Col HW, Kimsey CD, et al. Prevalence of physical activity, including lifestyle activities among adults. Weekly. 2003;52(32):764-9. 